Michel Grossetti

\title{
Logiques sociales et spatiales de la création d'entreprises innovantes
}

Géographie, Economie, Société, Vol. 10, n¹, pp. 5-7.

\section{Présentation du dossier}

La création d'entreprises innovantes est un leitmotiv des politiques de développement économique local. Les modèles plus ou moins fantasmés de la Silicon Valley ou des «clusters » américains de biotechnologies orientent de nombreuses actions publiques et envahissent les discours de «décideurs» de tous ordres. Pour les chercheurs en économie régionale ou en géographie industrielle, c'est aussi une question importante : on remplirait des immeubles entiers avec les publications consacrées aux «start-ups », aux « essaimages » et aux «clusters ». On sait déjà beaucoup de choses, comme le fait que les entreprises innovantes tendent à se concentrer dans l'espace, ou encore que celles qui sont essaimées des laboratoires de recherche s'installent en général à proximité de ceux-ci. Pourquoi dans ce cas, faire un dossier sur ce thème ? Parce que si l'on dispose de nombreux travaux en économie ou en géographie, la question a été peu traitée d'un point de vue sociologique. Or, les sociologues abordent le problème sous des angles un peu différents des disciplines précitées et ils conduisent des enquêtes empiriques d'une façon spécifique, ce qui permet de renouveler le regard sur le phénomène. Ils s'efforcent en particulier de comprendre les logiques des fondateurs d'entreprises, les raisons qui les amènent à se lancer dans le processus de création, la façon dont ils mobilisent leurs proches ou d'autres relations, leurs choix de localisation, leur insertion dans des milieux professionnels locaux ou non, dans des réseaux. A l'acteur économique parfois un peu désincarné, ils substituent un acteur social saisi dans l'épaisseur de ses multiples registres d'activité, engagé dans des relations et des collectifs multiples.

Les articles rassemblés dans ce dossier, dont plusieurs sont issus du programme «Les logiques spatiales de l'innovation ${ }^{1}$, intègrent tous, au moins pour partie, une perspective sociologique, et reposent tous sur des enquêtes de terrain approfondies, le plus souvent par entretiens. Erwan Lamy pose le problème du rôle de la proximité spatiale dans le cas d'entreprises créées en France par des chercheurs académiques. S'inscrivant dans une perspective de sociologie des sciences, il a interrogé 41 chercheurs ayant créé des entreprises, ce qui lui permet de distinguer différentes figures du chercheur entrepreneur, dont l'une seulement cherche à faire disparaître les frontières, tant sociales que physiques, entre la recherche et l'industrie. Pour les autres, ces frontières ne sont pas des obstacles aux échanges, elles les favorisent mêmes en permettant aux chercheurs de jouer sur plusieurs registres. La proximité spatiale entre le laboratoire d'origine et l'entreprise n'obéit dont pas nécessairement à la même logique selon les cas, et n'est le plus souvent pas nécessaire aux échanges. Fabien Reix s'est lui aussi intéressé à des entrepreneurs, mais qui ne sont pas nécessairement engagés dans des projets innovants. A partir de 45 entretiens biographiques avec

\footnotetext{
${ }^{1}$ Programme de Maison des Sciences de l'Homme d'Aquitaine, animé par Christophe Bounneau et Yannick Lung.
} 
des créateurs d'entreprises, il fait ressortir les effets sur l'engagement dans le processus de création de la trajectoire antérieure des créateurs, de leurs origines sociales, de leurs relations. Il montre en particulier que la création d'entreprise est un processus de découplage, qui s'ancre au départ dans des liens forts, très locaux, mais mobilise par la suite plus de liens faibles. La même idée et le même résultat ressortent de l'enquête que nous avons réalisée avec Jean-François Barthe et Christophe Beslay sur 54 histoires de créations d'entreprises « innovantes » de la région Midi-Pyrénées et des régions limitrophes au moyen d'entretiens croisés avec plus d'une centaine de fondateurs. L'enquête met en avant le caractère souvent collectif de l'activité de création, l'importance des relations sociales (l'encastrement si l'on préfère) dans l'accès aux ressources, notamment dans la phase de démarrage, et le fait que le choix de localisation s'effectue en général par défaut (on crée l'entreprise là où l'on vit). Un constat similaire sur les choix de localisation émerge de l'enquête de Claire Champenois sur 41 entreprises des «clusters » allemands de biotechnologie, mais elle insiste plus sur le poids des dispositifs de soutien à la création d'entreprise que sur les relations personnelles des fondateurs. Tout se passe comme si ces dispositifs se substituaient en partie à des réseaux interpersonnels, ce que l'on peut lire peutêtre, au-delà des différences de méthodologie avec les études sur le Sud-Ouest français, comme l'indice d'une efficacité plus grande des dispositifs mis en place en Allemagne. Enfin, Delphine Mercier et Jacques Garnier montrent comment s'enchaînent les générations d'entreprises avec l'étude comparée des entreprises créées par des anciens de Gemplus dans l'agglomération marseillaise et des nouvelles sociétés liées aux activités maritimes installées dans les anciens chantiers navals de La Ciotat. Leur étude met en évidence les effets d'emboîtement de contextes multiples, qui vont des trajectoires personnelles aux milieux professionnels, en passant par des niveaux d'action intermédiaires dont la nouvelle entreprise elle-même. Ils insistent en particulier sur la tension existant entre les différents niveaux d'action auxquels les fondateurs peuvent se référer et sur la reconfiguration permanente au fil du temps des ressources ou contraintes issues des différents niveaux.

Malgré la variété des terrains et des perspectives, les enquêtes sur lesquels s'appuient ces articles convergent très largement sur un certain nombre de points. Les auteurs sont unanimes sur les choix de localisation : ils ne sont qu'exceptionnellement gérés de façon stratégique et se font le plus souvent par défaut, ce qui questionne beaucoup de travaux entièrement fondés sur des hypothèses relatives aux stratégies de localisation. Ensuite, tous insistent sur l'importance des relations interpersonnelles et des réseaux sociaux, à l'exception peut-être de Claire Champenois, qui en atteste la présence, mais considère qu'ils sont secondaires par rapport aux dispositifs publics dans les clusters qu'elle a étudiés. On retrouve la thèse classique de l'encastrement des activités économiques dans les réseaux, mais les études présentées montrent aussi que l'encastrement est en tension avec diverses formes de découplage. Les dispositifs publics par exemple, permettent aux fondateurs de trouver des appuis lorsque leurs relations ne les leur procurent pas, découplant ainsi le processus de création des réseaux des fondateurs. Par ailleurs, la création de l'entreprise elle-même peut être vue comme un processus de découplage par la création d'un acteur collectif nouveau. Dans tous les cas, la création d'entreprises apparaît comme une activité fortement située, socialement et spatialement, qui ressemble moins à une activité purement stratégique d'optimisation des ressources et des contraintes, qu'à la recherche d'un équilibre précaire en faisant flèche de tout bois. C'est donc avant tout la répartition dans l'espace des fondateurs et des ressources qui rend compte des logiques spatiales que l'on peut observer. 
